\title{
Editorial: Grenzenlose Werbung zwischen Konsum und Audiovision
}

Wo endet Werbung, wo beginnt sie? Wie gestaltet sich die grenzenlose und vielschichtige Verbreitung und Rezeption der Werbung in Vergangenheit und Gegenwart? Wie haben sich Konsumkulturen verändert? Mit welchen Strategien versucht die Werbung, Emotionen und Verhaltensmuster zu generieren? Welche künstlerischen Entgrenzungen und konsumkritischen Überlegungen lassen sich anhand von Werbefilmen, -platten, TV-Programmen und interaktiven, digitalen Plattformen festmachen? Der vorliegende Band stellt sich diesen Fragen, versammelt vielfältige Beiträge zur Geschichte audiovisueller Werbung und fokussiert dabei auf Grenz- und Sonderfälle, Zwischen- und Übergangsformen sowie Transformationsphasen der Werbegeschichte. Angeknüpft wird an Forschungen, die in den letzten Jahren vermehrt das komplexe Verhältnis von Werbung, Konsumkultur und audiovisuellen Medien in den Blick nahmen. Ein Verhältnis, das lange Zeit wenig beforscht wurde und $\mathrm{zu}$ dem erst in den letzten Jahren umfassendere Studien entstanden. ${ }^{1}$ Dabei wurde auch die in der bisherigen Werbegeschichtsschreibung implizite modernistische Sichtweise hinterfragt, nach der Werbung als ein zunehmend an Macht, Einfluss und Professionalität gewinnendes System betrachtet wurde. ${ }^{2}$ Dessen Dekonstruktion führte zu einem wachsenden Interesse an der Heterogenität von Werbeformen und dem Spannungsfeld zwischen den Ansprüchen der Konsument*innen, den Möglichkeiten der Medien und den Absatzinteressen der Unternehmen. Wir haben es also nicht mit einer linearen Erfolgsgeschichte zu tun, sondern mit einer Entwicklung, die durch zahlreiche Grauzonen, kurz- und langfristige Irrwege, erfolgreiche und gescheiterte Experimente, insgesamt von einem beständigen Veränderungs- und Innovationsdrang gekennzeichnet war. ${ }^{3}$ Wie die aktuelle Forschung zeigt, bedienten sich Werber`innen im Lauf der Geschichte aller nur erdenklicher medialer Formen, von denen manche heute in Vergessenheit geraten sind. Eindrucksvoll vor Augen führen dies Solveig Ottmann und Sandra Reimann in diesem

\footnotetext{
1 Vonderau, Patrick, Bo Florin u. Nico de Klerk (Hrsg.): Films That Sell. Moving Pictures and Advertising. London 2017; Moser, Karin: Der österreichische Werbefilm. Die Genese eines Genres von seinen Anfängen bis 1938. Berlin/Boston 2019: http://library.oapen.org/bitstream/id/299047985d01-44b8-9369-3513a5034a50/1006587.pdf (25.4.2020); Grainge, Paul u. Catherine Johnson: Promotional Screen Industries. Hoboken 2015.

2 Vonderau, Patrick: Introduction: On Advertising's Relation to Moving Pictures. In: Films That Sell. Hrsg. von Vonderau, u.a. (wie Anm. 1), S. 3; McFall, Liz: Advertising. A Cultural Economy. London 2004. S. 189.

3 Vgl. Vonderau, Introduction (wie Anm. 2), S. 3.
} 
Band mit einem Beitrag zur Geschichte der Werbeschallplatte, einem Werbemedium, das trotz seiner einst großen Verbreitung, bisher kaum erforscht wurde.

Entstanden ist der vorliegende Band als Resultat der Tagung Werbung - Konsum - Audiovision. Kulturgeschichtliche Perspektiven auf Werbeformen in Kino, Fernsehen und Radio, die im November 2017 in Wien stattfand. ${ }^{4}$ Mit der von den Herausgeber`innen organisierten Veranstaltung galt es insbesondere auch den wissenschaftlichen Austausch zwischen den Disziplinen zu befördern. Gerade bei einem Forschungsgegenstand wie der Werbung, der bekanntlich von unterschiedlichen kultur- und sozialwissenschaftlichen Disziplinen beforscht wird, sollte das interund transdisziplinäre Gespräch unerlässlich sein. Ziel der Tagung war es darüber hinaus, aktuelle Trends und Tendenzen in der Erforschung audiovisueller Werbeformen auszuloten. Die erfreulich große Reaktion auf den Call for Papers zeigte, dass sich Studien zur Geschichte und Gegenwart der Werbung nicht auf audiovisuelle Werbeinhalte und -medien als solche beschränken, sondern vermehrt nach den strukturellen Bedingungen und Kontexten von Unternehmen, Medien und der Werbebranche gefragt wird. Ging es bislang primär um eine Analyse der (Konsum-)Ge-

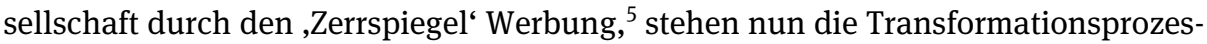
se von Werbeformaten, der Werbebranche sowie der Zusammenhang dieser mit dem sozioökonomischen Wandel im Fokus. ${ }^{6}$ Diese Perspektive zeigte sich bei der Tagung als gemeinsamer Schnittpunkt der Tagungsteilnehmerinnen und animierte die Herausgeber*innen dazu, diesen Band zur Grenzenlosen Werbung zu publizieren.

Die hier versammelten Beiträge widmen sich erstens der Untersuchung von Transformationsphasen in der Geschichte der Werbung. So behandeln Dirk Schindelbeck und Anne Schmidt Fallbeispiele aus der Frühphase des Werbefilms und der Umbruchsphase der Zwischenkriegszeit. Die Beiträge von Bernhard Dietz und Mario Keller untersuchen anhand des Camel-Man und der Avantgardekunst-Werbung von

4 Vgl. den Tagungsbericht von Alexander Obermüller, https://www.hsozkult.de/conferencereport/ id/tagungsberichte-7536 (26.3.2020). Die Tagung erfolgte im Rahmen des von den Veranstalter^innen durchgeführten FWF-Forschungsprojektes „Die Emotionalisierung nationaler Marken im österreichischen Werbefilm 1950-2000“, vgl. https://oesterreichischer-werbefilm.univie.ac.at (26.3.2020).

5 Vgl. ältere Klassiker der Werbegeschichte wie: Marchand, Roland: Advertising the American Dream. Making Way for Modernity, 1920-1940. Berkeley 1986; Gries, Rainer, Volker Ilgen u. Dirk Schindelbeck: „Ins Gehirn der Masse kriechen!“. Werbung und Mentalitätsgeschichte. Darmstadt 1995; Schmidt, Siegfried J. u. Brigitte Spieß: Die Kommerzialisierung der Kommunikation. Fernsehwerbung und sozialer Wandel 1956-1989. Frankfurt a. M. 1997.

6 Vgl. Swett, Pamela E., S. Jonathan Wiesen u. Jonathan R. Zatlin (Hrsg.): Selling Modernity. Advertising in Twentieth-Century Germany. London 2007; Turow, Joseph u. Matthew P. McAllister (Hrsg.): The Advertising and Consumer Culture Reader. London 2009; Wharton, Chris: Advertising. Critical Approaches. Hoboken 2014; Wharton, Chris (Hrsg.): Advertising as Culture. Bristol 2013; Grainge u. a. (Hrsg.), Promotional (wie Anm. 1), u.v.m. 
Humanic den Einfluss der ,68er` auf die Entwicklung der Fernseh-Werbung. Zweitens werden vermeintlich randständige Medien, wie die schon erwähnte Werbeschallplatte von Solveig Ottmann und Sandra Reimann, in den Blick genommen. Drittens wird die Rolle staatlicher Institutionen und der von ihnen ausgehenden Vorgaben und Einschränkungen untersucht. Dies betrifft sowohl historische wie auch gegenwärtige Entwicklungen. So analysiert Sebastian Thalheim einen DDRWerbefilm für die Schmalfilmkamera $A K$, in dem neben dem Produkt auch die vermeintliche Überlegenheit der sozialistischen Konsumkultur beworben wurde. Karin Moser untersucht die Kino- und Fernsehwerbefilme des österreichischen staatlichen Tabak-Monopolkonzerns Austria Tabak. Während diese in den 1950er Jahren noch im Einklang mit der staatlichen Wiederaufbaupropaganda standen („Rauchen für Österreich“), wurden sie seit den 1980er Jahren zunehmend durch rechtliche Beschränkungen und Werbeverbote reglementiert. Rezente und in die Zukunft weisende Entwicklungen stehen im Mittelpunkt der Beiträge von Stephanie Scholz, Guido Zurstiege und Michael Cowan. Stephanie Scholz analysiert ,program length commercials“ im deutschsprachigen Fernsehen und kann anhand von ,Spielzeughelden“ im Kinderprogramm eine Verschmelzung von Programm und Werbung beobachten. Auch Guido Zurstiege diagnostiziert eine zunehmende Fusion von herkömmlichen Inhalten und werbenden Botschaften im Rahmen des digitalen Medienwandels. Michael Cowan zeigt anhand des dystopischen Spielfilms Minority Report, dass die dortigen interaktiven bzw. reaktiven Werbeformen sowie Bedürfnis- und Wunschprognosen bereits Realität geworden sind.

Mit Blick in die Vergangenheit, Gegenwart und Zukunft werden in diesem Band auch die Grenzen der Werbung reflektiert: rechtlich bzw. staatlich, gesellschaftlich, ästhetisch, medial sowie strukturell und institutionell. In der Einleitung des 2002 erschienen Sammelbandes Die Gesellschaft der Werbung behauptete Herbert Willems, dass Werbung „normalerweise“ mit offenen Karten spiele und sich als solche zu erkennen gebe. Zwar wies er auch darauf hin, dass die Bandbreite der Irreführungen, Täuschungen und Verschleierungen zunehme, sich die Mehrheit der Werbungen jedoch innerhalb eines „offiziellen Rahmens“ bewege. ${ }^{7}$ Doch wo beginnt und wo endet dieser ,Rahmen“ und von wem wird er definiert? Die vorliegenden Studien legen jedenfalls die Vermutung nahe, dass die Geschichte der Werbung dadurch gekennzeichnet ist, dass die Norm bzw. Normalität des jeweiligen Rahmens ausgereizt, überdehnt oder gar bewusst gesprengt wird, um immer wieder neue Aufmerksamkeit generieren zu können. So gesehen war und ist Werbung einem ständigen Aushandlungsprozess unterworfen und damit ein hochpolitisches Medium, in dem die Macht und Ohnmacht von Produzent*innen und Konsument*innen - oft

7 Willems, Herbert: Grundideen des Bandes. In: Die Gesellschaft der Werbung. Kontexte und Texte. Produktionen und Rezeptionen. Entwicklungen und Perspektiven. Hrsg. von Herbert Willems. Wiesbaden 2002. S. 17-18. 
auch von Prosumer*innen und Konduzent*innen - permanent repositioniert wird. ${ }^{8}$ Die gegenwärtige, bisher ungeahnte Konzentration von Medienmacht in den Händen der vier dominierenden US-amerikanischen Digitalkonzerne Alphabet/Google, Amazon, Facebook und Apple sowie des chinesischen Hyper-Konzerns Alibaba Group war nur möglich, weil diese mehr und mehr Werbeeinahmen von den klassischen Medien abziehen können. Heute geben diese Konzerne immer häufiger den Rahmen vor, in dem sich die Werbung bewegt und nicht mehr staatliche Akteur ${ }^{\star}$ innen und Institutionen. ${ }^{9}$

Publikationen zur Geschichte der Werbung beginnen häufig mit der Feststellung, dass Werbung so alt sei wie die Menschheit selbst. ${ }^{10}$ Aus (geschichts-)wissenschaftlicher Sicht scheinen solche Aussagen jedoch beliebig und wenig gewinnbringend. Vielmehr sollte die Geschichte der Werbung zu jenem Zeitpunkt ansetzen, an dem sich die persuasive Kommunikation über Produkte professionalisierte und zu einem Wirtschaftszweig entwickelte. So gesehen begann die Geschichte der Werbung als selbstständige Wirtschaftsbranche erst in der zweiten Hälfte des 19. Jahrhunderts. Die Entwicklung der Werbewirtschaft war dabei aufs Engste mit der Entstehung der Konsumindustrie, der Konsumgesellschaft und dem Erfolg ,neuer Massenmedien verknüpft. Dieses Verständnis von Werbe-Wirtschaft als einem konstanten und ambivalenten Spannungsverhältnis zwischen Unternehmen, Konsument*innen und Medien beugt einem affirmativen und/oder fatalistischen Verständnis von Werbung vor. Hier geht es nicht darum, Werbung als Manipulationsmaschine zu verteufeln oder umgekehrt als unverzichtbaren, weil die Nachfrage stimulierenden, Wirtschaftsmotor zu bejubeln. Vielmehr sollte Werbung als Wirtschaftszweig und kulturelle Praxis beschrieben und analysiert werden, deren Expertise darin besteht, ökonomischen Nutzen aus der Kommunikation von kulturellen Vorstellungen mittels emotionalisierender Strategien zu ziehen und damit Aufmerksamkeit und Öffentlichkeit für Produkte und Marken zu generieren. ${ }^{11}$

Werbetreibende setzten schon immer unterschiedliche mediale Kanäle ein. Auch Film, das erste audiovisuelle Massenmedium, wurde von Beginn an für werbe-

8 Siehe dazu das Schwerpunktheft der ÖZG. Österreichische Zeitschrift für Geschichtswissenschaften 30 (2019/1) mit dem Titel „Produzieren/Konsumieren - Prosumieren/Konduzieren“.

9 Brodnig, Ingrid: Übermacht im Netz. Warum wir für ein gerechtes Internet kämpfen müssen. Wien 2019. S. 41-57; Fuchs, Christian: Culture and Economy in the Age of Social Media. New York 2015.

10 Prominent vertreten wurde diese These von Hanns Buchli. Vgl. Buchli, Hanns: 6000 Jahre Werbung. Geschichte der Wirtschaftswerbung und der Propaganda. Band 1-3. Berlin 1962; vgl. auch Denscher, Bernhard: Kunst \& Kommerz. Zur Geschichte der Wirtschaftswerbung in Österreich. Wien 1985.

11 Zur Theorie des Systems Werbung siehe insb. die Arbeiten von Guido Zurstiege. Vgl. u. a. Zurstiege, Guido: Zwischen Kritik und Faszination. Was wir beobachten, wenn wir die Werbung beobachten, wie sie die Gesellschaft beobachtet. Köln 2005; Zurstiege, Guido: Medien und Werbung. Wiesbaden 2015. 
rische Zwecke verwendet und auf vielfältige Weise genutzt. ${ }^{12}$ Der vorliegende Band widmet sich vor allem, aber nicht ausschließlich, der Geschichte audiovisueller Medien - konzise Überlegungen zu den Spezifika dieser Mediengattung sind also notwendig. Unter audiovisuellen Werbemedien werden alle Medienformate verstanden, die als Kombination von Bild, Ton und/oder Text, und somit als multisensuale und multimodale Medien in Erscheinung treten. Multisensual sind sie, da Medienrezipient*innen stets auf mehreren Sinnesebenen adressiert werden; multimodal, da diese Sinnesebenen mittels unterschiedlicher Modi angesprochen werden. Auf der auditiven Ebene sind dies beispielsweise Geräusch, Musik und sprachliche Artikulation. ${ }^{13}$ Das Zusammenwirken dieser sich ergänzenden und bedingenden Kommunikationswege und -strategien ist somit für jede Analyse audiovisueller Medienprodukte unerlässlich. Zweifellos eröffnen die Multimodalität und Multisensualität audiovisueller Medien unüberschaubare Möglichkeiten, Emotionen zu kommunizieren. ${ }^{14}$ Aus Sicht von Werbetreibenden werden Produkte und Marken auf diesem Wege mit emotionalem und kulturellem Mehrwert bzw. Zusatznutzen konnotiert und assoziiert. Auch wenn die emotionalisierende Dimension bei der Analyse audiovisueller Werbung (und audiovisueller Medien allgemein) auf der Hand liegt und für Werbetreibende eine zentrale Rolle spielt, wurde diese in der kulturwissenschaftlichen Werbeforschung bisher kaum berücksichtigt. In diesem Sammelband zeigt zum Beispiel Anne Schmidt wie in Werbefilmen der Zwischenkriegszeit bewusst mit der Evokation von Angstgefühlen gearbeitet wurde. Karin Moser erläutert, wie in den Werbefilmen der Tabakindustrie die Grenzen zu populären Spielfilmen und Musikvideos verschwimmen und wie über filmische Mittel und kulturelle Assoziationshöfe Spannung bzw. Konzentration generiert und Emotionen frei gesetzt werden. Ansätze der Emotionsgeschichte, der neueren Werbegeschichte sowie der transdisziplinären Medien- und Filmwissenschaften werden dazu führen, dass sich die (historische) Werbeforschung vermehrt mit der Rolle von Emotionen in audiovisuellen Erzählstrategien befasst. ${ }^{15}$

Ein weiteres Spezifikum von audiovisuellen Werbungen sei abschließend genannt. Wie bei der Produktion und Distribution ist auch bei der Archivierung von

12 Moser, Der österreichische Werbefilm (wie Anm. 1): Vonderau, Introduction (wie Anm. 2), S. 4. 13 Stöckl, Hartmut: Hörfunkwerbung - „Kino für das Ohr“. Medienspezifika, Kodeverknüpfung und Textmuster einer vernachlässigten Werbeform. In: Textdesign und Textwirkung in der massenmedialen Kommunikation. Hrsg. von Kersten Sven Roth u. Jürgen Spitzmüller. Konstanz 2007. S. 183. 14 Vgl. Bartsch, Anne, Jens Eder u. Kathrin Fahlenbach (Hrsg.): Audiovisuelle Emotionen. Emotionsdarstellung und Emotionsvermittlung durch audiovisuelle Medienangebote. Köln 2007. Intensiv mit der Verwobenheit von Kognition, Emotion und filmischer Erzählung befasst sich die, v. a. im englischsprachigen Raum etablierte Schule der cognitive film studies. Vgl. Smith, Greg M. u. Carl Plantinga (Hrsg.): Passionate Views. Film, Cognition, and Emotion. Baltimore 1999; Smith, Greg M.: Film Structure and the Emotion System. Cambridge 2003. u.v. m.

15 Vgl. Plamper, Jan: Geschichte und Gefühl. Grundlagen der Emotionsgeschichte. München 2012. S. 334 . 
audiovisuellen (Werbe-)Medien ein vergleichsweise hoher technischer Aufwand notwendig. Im Archiv erfordern audiovisuelle Medien ebenfalls eine aufwendige und teils komplizierte ,Behandlung‘. Ihre Bewahrung und Erschließung ist aus diesem Grund meist prekärer als beispielsweise bei Printmedien und Plakaten. Audiovisuelle Werbemedien wurden oftmals nicht gesammelt oder als erhaltenswert erachtet. Sie waren kurz und flüchtig im Einsatz (,ephemer“). Hatten sie ihren Zweck erfüllt, wurden sie vergessen („orphan“). Die heutige Vielzahl an audiovisuell verfügbaren Werbemedien im Internet vermittelt eine vermeintliche ,totale Zugänglichkeit‘. Ob diese digitalen Quellen auch in Zukunft abrufbar sein werden, ist nicht gesichert. Allen voran kommen hier rechtliche Fragen zum Tragen, die den Zugang und die Nutzung weniger ent- als begrenzen. Da Werbehistoriker*innen mit dieser Situation konfrontiert werden, haben wir einen Beitrag von Gabriele Fröschl, der Archivleiterin der Österreichischen Mediathek, aufgenommen, der diese Problematik umfassend beleuchtet.

Zuletzt sei den fördergebenden Institutionen sowie den an der Tagung und der Entstehung des Bandes beteiligten Personen gedankt. Die Abhaltung der Tagung wurde vom österreichischen Fonds zur Förderung der wissenschaftlichen Forschung (FWF), dem Forschungsschwerpunkt Wirtschaft und Gesellschaft aus kulturwissenschaftlicher Perspektive der Historisch-Kulturwissenschaftlichen Fakultät der Universität Wien, der Vienna Doctorial Academy - Theory and Methodology in the Humanities, dem Institut für Wirtschafts- und Sozialgeschichte sowie der Historisch-Kulturwissenschaftlichen Fakultät der Universität Wien finanziell unterstützt. Weiters gedankt sei Veronika Haydn, Johann Kirchknopf, Oliver Kühschelm, Stefan Ossmann und Alexander Obermüller für ihre tatkräftige Unterstützung bei der Durchführung der Tagung. Die Drucklegung des Bandes war nur durch eine Subvention der Historisch-Kulturwissenschaftlichen Fakultät der Universität Wien sowie einen finanziellen Beitrag der Österreichischen Mediathek und des Forschungsschwerpunkts Wirtschaft und Gesellschaft aus historisch-kulturwissenschaftlicher Perspektive der Historisch-Kulturwissenschaftlichen Fakultät der Universität Wien möglich. 